\title{
Extending, mapping, and focusing the CIDOC CRM
}

\author{
Introduction to the special issue
}

\author{
Franco Niccolucci $^{1}{ }^{[}$
}

(C) Springer-Verlag Berlin Heidelberg 2016

\section{Introduction}

The present special issue of IJDL is dedicated to extensions and specializations of the CIDOC CRM [1], the well-known Conceptual Reference Model for the documentation of cultural heritage.

The CRM is programmatically extensible, i.e., it allows defining new classes and properties on top of the core model to create a domain ontology, more suitable for the knowledge organization of that specific domain. Such CRM extensions are subject to a double validation process. On the one hand, domain experts propose or comment the new classes and properties introduced in the extension, in light of the domain documentation needs. On the other hand, the extension proposal is discussed from a logical and technical perspective by the CRM special interest group (SIG), both online and during its biannual meetings, until consensus is reached. The extension is then tested in practical applications to verify in the field its suitability for the purpose. The extension technical documentation is posted as a technical report on the CRM web pages, hosted at FORTH [2]. Such extensions are a sort of formally accepted ones, but of course, everybody is free to create their own model and use it, since all CRM documents are openly accessible.

While this process guarantees the formal logical coherence of the proposed extension and its suitability to a specific domain, it risks that the extension is well known only by a limited number of researchers. The extension authors usually report about their work at heritage-related conferences, for example, as regards archaeology, the Computer Applications

Franco Niccolucci

franco.niccolucci@gmail.com

1 PIN-Polo Universitario di Prato, Piazza Ciardi 25, 59100 Prato, Italy in Archaeology (CAA) yearly conference, but the audience is here more interested in the use of the extended schema than in its architecture, logical structure and formal coherence. On the other hand, technical conferences often do not have the necessary interest and experience in domain issues motivating the creation of new classes and properties.

Thus, since a few years the International Conference on Theory and Practice of Digital Libraries (TPDL) hosts a workshop titled CRM Experiences (CRMEX) dedicated to CRM-related topics, where presentations of new extensions are presented together with reports of activities using the CRM. At TPDL2015 in Poznan, this workshop collected several presentations about the most recent advances in the CRM extension process, suggesting that the topic might be interesting and have enough material for a dedicated issue of a specialized journal such as IJDL. In the journal editors' intention, this special issue might provide a consolidation of the current advancements; it might further validate results through peer review and reach a wider audience, such as the journal one, spreading the knowledge about the current CRM progress and stimulating new contributions. Thus, they asked me, as the main organizer of the TPDL2015 workshop, to serve as guest editor of this issue. The call for papers circulated in late 2015 got enough interesting material for the special issue, which has now completed its revision cycle and appears with eight articles. All the papers published here are either completely new ones, or revised and extended versions building on the original TPDL2015 presentations.

The papers of the present special issue may be grouped in three sets.

The first one consists of papers about some new or consolidated CRM extensions needing a formal assessment and description. Each of them includes a discussion of the domain issues pushing the need for an extension and why the solution presented was chosen. This group includes the paper by 
Ronzino on the CRMba extension, addressing the documentation needs of archaeological standing structures, and how this extension may be harmonized with CRMarcheo, another CRM extension for the archaeological domain. Felicetti's and Murano's paper deals with epigraphy, discussing the issues of documenting inscriptions, complex objects incorporating both a physical and a conceptual component, respectively, the support and the content of the inscription, so far managed separately in most cases. A CRM extension titled CRMtext is then proposed for this purpose. The next paper in this group, by Hiebel, Doerr, and Eide, describes CRMgeo, a CRM extension concerning the spatiotemporal properties of temporal entities and persistent items, but also reconciling the CRM with the schema proposed by the Open Geospatial Consortium (OGC) in GeoSPARQL. The last paper of the group is the one by Niccolucci and Hermon, discussing ways to deal with fuzzy information and proposing a minimal addition of classes and properties to document uncertain interpretation.

This paper includes an extended discussion about reliability, and thus, it also relates to the second group dedicated to a reflexive discussion of open issues, which, furthermore, includes the paper by Guillem, Bruseker, and Ronzino about architectural concepts and how to deal with them. Both papers are characterized by the ample space dedicated to critically motivate the need for an extension.

The third and last group of papers describes specific CRM applications or tools. It includes the paper by Marketakis et al., describing a novel mapping tool from metadata schemas to CRM concepts, necessary to integrate legacy data sets into a global CRM framework. This tool has been tested in practice with excellent results. In addition, the paper by Velios and Martin provides a tool, in this case, a method to use Drupal to set up a CRM-based web environment. It also presents an application to decorated papers. Finally, the last paper, by Mäkelä, Törnroos, Lindquist and Hyvönen concerns an application of the CRM to WW1 documents, discussing specific issues related to this theme.

The editor of this special issue hopes that the contributions presented here stimulate other researchers in the field of knowledge engineering and organization to further investigate this topic. In the era of Open Data and Open Science, openness without a common logical structure risks to generate just more confusion. An extensible ontology such as the CRM is the perfect tool to organize heritage-related knowledge, as it conjugates the rigidity of a standard, enabling robust data integration, with the flexibility of application-led schemas, supporting all the diverse needs of research in the multifaceted domain of cultural heritage and humanities.

\section{References}

1. CIDOC CRM: Current Official Version of the CIDOC Conceptual Reference Model. Version 6.2 of the reference model. http://new. cidoc-crm.org/node/202 (2016). Accessed Oct 2016

2. CIDOC CRM: Technical documents: http://new.cidoc-crm.org/ technicalpapersPage (2016). Accessed Oct 2016 